\title{
Continuous plasma density measurement in TJ-II infrared interferometer-Advanced signal processing based on FPGAs
}

\author{
L. Esteban ${ }^{\mathrm{a}, *}$, M. Sánchez ${ }^{\mathrm{a}}$, J.A. López ${ }^{\mathrm{b}}$, O. Nieto-Taladriz ${ }^{\mathrm{b}}$, J. Sánchez ${ }^{\mathrm{a}}$ \\ - Laboratorio Nacional de Fusión, Asociación EuRAToM-CIEMAT, Avenida Complutense No. 22, E-28040 Madrid, Spain \\ b Departamento de ingeniera Electronica, ETSI Telecomanicacton, Universidad Poiltcnica de Madrid, Avenida Complitense No. 30 , E-28040 Madrid, Spain
}

Keywords:

FPCAA

DSP

Multicliannel intertètometry

Data acquisition

Realtime

Phasemeters

\begin{abstract}
A B S T R A C T
This work presents the behavioral simulation in an FPGA of a novel processing system for measuring line average electronic density in the TJ-II stellarator diagnostic, Infra-Red Two-Color Interferometer. Line average electronic density is proportional to phase difference between probing and reference signals of the interferometer, as the Appleton-Hartree cold plasma model states. The novelty of the approach is the development of a real time measuring system where research work has been carried out in two ways: a new interpolation algorithm and the implementation of a new specific processor on an FPGA.

The main goal of this new system is to measure line plasma electronic density for several channels in real time, also it will be useful to eliminate intermediate mixing frequency stages (the output signals coming from the interferometer are going to be directly sampled) and finally to generate real time density signals for control purposes in TJ-Il and in other diagnostics. This device is intended to be the new data acquisition-processing system for the future six channel infrared interferometer that requires at least 14 input signals. The knowledge acquired could be useful in the design of W7-X and ITER IR-interferometer data acquisition and processing systems.
\end{abstract}

(c) 2010 Elsevier B.V. All rights reserved.

\section{Introduction}

The TJ-II stellarator [1] has a two color one channel Mach-Zehnder heterodyne interferometer, one color $(10.6 \mu \mathrm{m} \mathrm{CO}$ laser) is used to measure plasma density and the other color (1.06 $\mu \mathrm{m}$ NdYAG laser) to correct mechanical vibrations [2], the two interferometers share the same optical path. The carrying frequencies are about $40 \mathrm{MHz}$. Each of these interferometers has two arms, one is used as a reference beam and the other one crosses the plasma. When both beams reach the correspondent detector an interference signal is generated. Line average electronic plasma density is:

$\int n_{\mathrm{e}} \mathrm{d} l=n_{\mathrm{c}} \mathrm{CO}_{2} \frac{\Delta \varphi \mathrm{NdYAC} \lambda_{\mathrm{N} d Y A C}-\Delta \varphi_{\mathrm{CO}_{2}} \lambda_{\mathrm{CO}_{2}}}{2 \pi}$

So phase measurement should be achieved for the four signals. The system used till now down-converts the interferometer signals by the use of an intermediate frequency mixing stage $(40 \mathrm{MHz}$ to $1 \mathrm{MHz}$ ), these signals are under-sampled and stored as raw data. After the acquisition the electron density is computed off-line. A new system to acquire and compute the line average electronic density with online processing capability and direct sampling of the interferometer signals is under development and to be installed in TJ-II experimental hall in next campaign. The expertise acquired will be translated to W7-X Stellarator [R-Interferometer [3].

\section{The online processing concept}

The purposes of this project are:

1. To obtain real time signals for control feedback.

2. To reduce noises and sources of error such as crosstalk [5], frequency instabilities and quantification errors, due to fixed point implementation of the algorithm in the FPGA [6].

3. To detect and eliminate bias (thermo-optical effect [7]) drift in steady state pulses (W7-X, ITER).

4. To manage all the signals of multi-channel systems in one unique board.

To achieve these goals, signals from the interferometer channels are fed at the input of the system and pre-processed to de-noise the carriers as seen in Fig. 1.

In the signal pre-processing block the input signals are de-noised from: crosstalk between the four channels, intermodulation generated in the detectors and amplifiers, noise due to the sub-sampling procedure if applicable and other sources of 


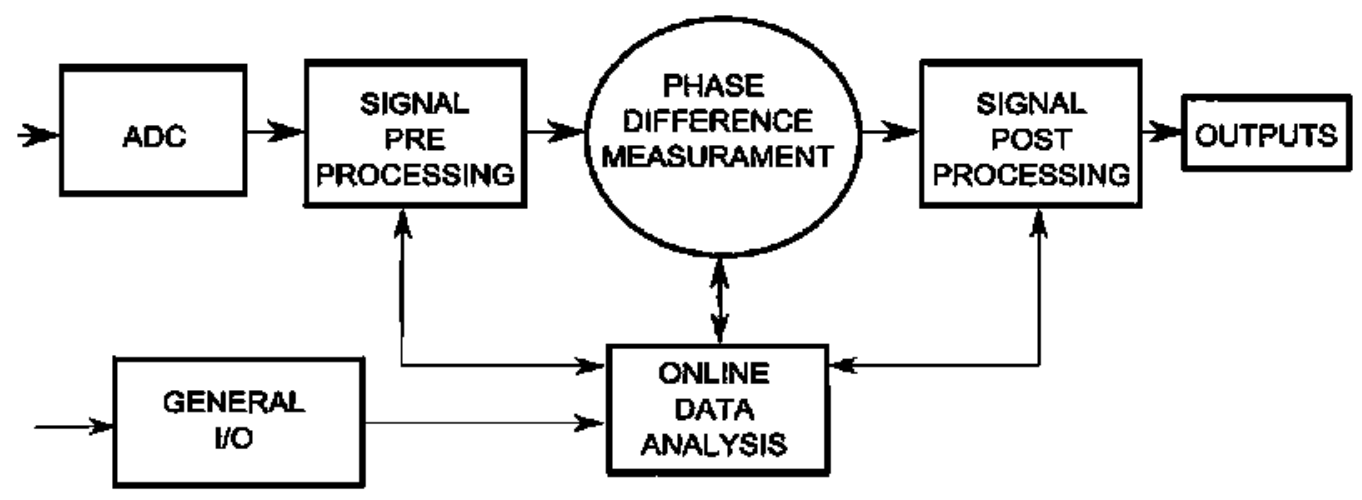

Fig. 1. Online processing block diagram.

causal noises coupled into the carriers. The bandwidth of random noise is also reduced. In this step we can diplex the signals if the same detector is used for the two colors, and carrier frequencies are separated enough ( $40 \mathrm{MHz} \mathrm{CO}_{2}$ and $25 \mathrm{MHz} \mathrm{CO}, \mathrm{W} 7-\mathrm{X}$ ). The filters should have a linear phase response and the same group delay for all the signals. This last task can be done with this system much more easily than with analog filters.

The signals coming from other diagnostics can be used to correct dependencies of others parameters at this stage. Afterwards phase difference is calculated with an original phase measurement algorithm developed for this project and described in detail in next section.

Finally electronic density signal is post-processed to de-noise the output signal. Several effects like the thermo-optical dispersion variation in the ZnSe windows can be reduced using other signals from the machine. Other sources of time dependent dispersion variations could be also compensated (air heating, windows stress, etc.). A Fourier analysis will permit the detection of noncompensated low frequency vibrations. The use of wavelets to clean the output signal improves the classical filtering [8], so an online implementation of this procedure would also be useful. As the machine time constant is typically higher than $1 \mathrm{~ms}$, with an output refresh of the control signal lower than $1 \mathrm{ksps}$ would be enough, low frequency noise and bias must be suitably bounded in acceptable limits in the control signal. The interferometer phase noise has an $1 / \mathrm{f}^{2}$ dependence (random walk) so the low frequencies are the most difficult to eliminate without signal distortion. Special care should be taken in the determination of the acceptable noise bounds, also the study of correlations with other parameters of the machine could help in this task. ln this last stage a tomographic reconstruction can be implemented in multi-channel systems to obtain spatial distribution of electronic density [3]. The whole process will be controlled by the online data analysis block.

The algorithm is being implemented in a Virtex IV SX55 FPGA. The analog-to-digital converters used in this design are AD6645 from Analog Devices. Sixteen analog inputs (driven at a sampling rate of $104 \mathrm{MHz}$ ) are available now, seven optical channels can be measured and the number of inputs can be easily expanded. Such high speed ADCs are needed because the AOMs fix the output signals at approximately $40 \mathrm{MHz}$.

\section{Phase measurement algorithm}

The implemented algorithm is a modification of an original interpolation algorithm based in $\mathrm{AD}$ conversion being used in the T]-[] [R-interferometer [4] for several campaigns and illustrated in Fig. 2.

If $S_{j}$ and $S_{j+1}$ are the signal values of the previous and following samples of a zero cross Fig. 2 , and $\Delta t$ the sampling period, then time $\tau_{1}$ from the previous sample to the zero cross can be computed as:

$\tau_{\mathrm{n}}=\frac{\left|S_{j}\right|}{\left|S_{j}\right|+\left|S_{j+1}\right|} \Delta t$

The temporal position of the $n$ zero cross is then:

$t_{n}=i \Delta t+\tau_{\mathrm{n}}$.

where $i$ is the sample index. The phase is:

$\psi_{i}=2 \pi n+\frac{2 \pi\left[(i-j) \Delta t-\tau_{n}\right]}{t_{n+1}-t_{n}}$.

$T_{\mathrm{n}}$ is the current signal period:

$T_{n}=(k-j) \Delta t+\tau_{n+1}-\tau_{\mathrm{n}}$

Being $j$ the number of samples acquired immediately after the detection of a zero cross and $k$ the number of samples previous to the next zero cross. As seen in the calculation of $T_{n}$, future data is taken into account, $k$ and $\tau_{n+1}$, so the process is non causal. Knowing the phase at each sample for both reference $\varphi_{\mathrm{ri}}$ and measuring $\varphi_{m i}$ signals the computation of the phase difference is direct:

$\Delta \varphi=\varphi_{\mathrm{mi}}-\varphi_{\mathrm{ri}}$.

To implement this algorithm in an FPGA all the data should be known, so every reference to future data should be transformed into references to past data. $\tau_{n}$ is calculated now as:

$\tau_{\mathrm{n}}=\frac{\left|S_{j}\right|}{\left|S_{j}\right|+\left|S_{j-1}\right|} \Delta t$

And now the current period $T_{n}$ is not known so the period from the previous zero cross is used. Other approach could be to introduce a latency in the system. Because a resolution of $1 / 1000$ in each signal is needed to recover the electronic density with a precision of about $1 / 400$, averaging of the signals is essential. Another method

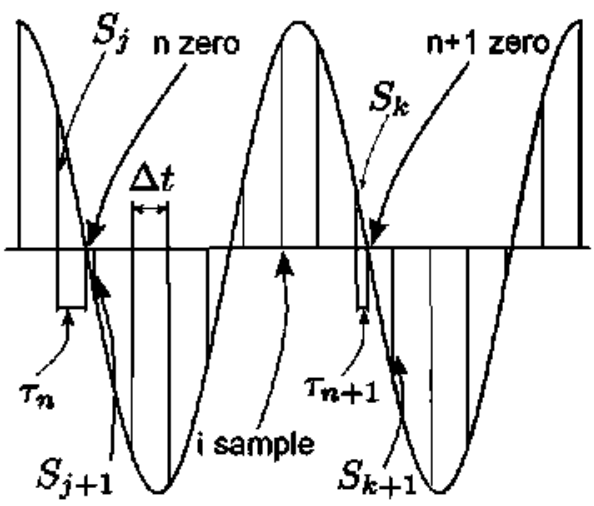

Fig. 2. Interpolat ion algoritlım. 


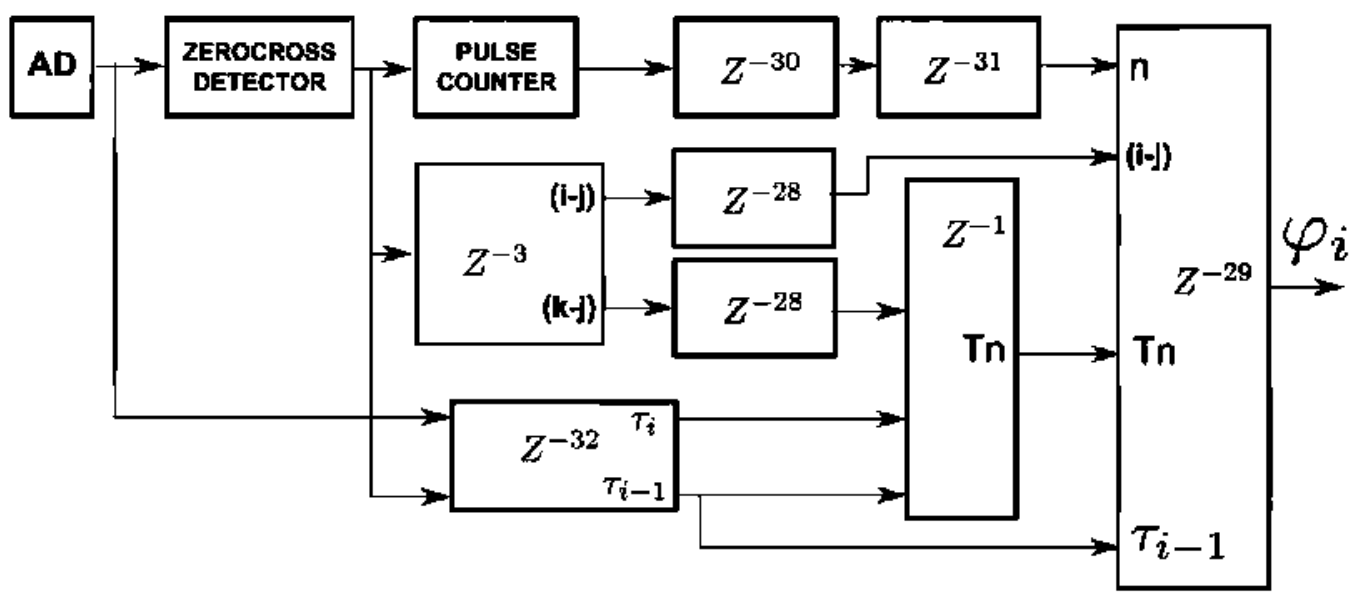

Fig. 3. Phase measurement data-path.

to measure phase difference is to just count zero crossings of the signals but the results obtained have much less resolution as it is detailed in next section.

In Fig. 3 part of the data-path is sketched:

The algorithm has been implemented in a pipelined way, several delay lines have been included to equalize data-paths. These delays are due mainly because two dividers are used to compute Eqs. (4) and (6). For this purpose standard XILINX cordic dividers have been used, future improvements of the design include the use of custom dividers.

The zero crossings are counted using a pulse counter and the $i-j$ and $k-j$ values, just with simple accumulators. Finally with all data equalized phase is computed (4). This procedure is done in paralle] in TJ-ll for the four signals and a substraction between the phases of probing and reference signals is done. After the substraction the phase difference signals are averaged and scaled $\mathrm{CO}_{2}$ signals are multiplied by the $\mathrm{CO}_{2}$ wavelength $10,591 \mu \mathrm{m}$ and same is done for NdYAG in this case by $1064 \mu \mathrm{m}$, finally subtracting these two quantities the average line electronic plasma density is obtained.

\section{Results}

Due to the impossibility to install the system in T]-[l experimental hall until next campaign, the phase measurement processor has been simulated with real data (and being implemented in an FPGA) acquired with the actual system. Signals from pulse 19033 have been frequency scaled to synthesize the conditions in which the

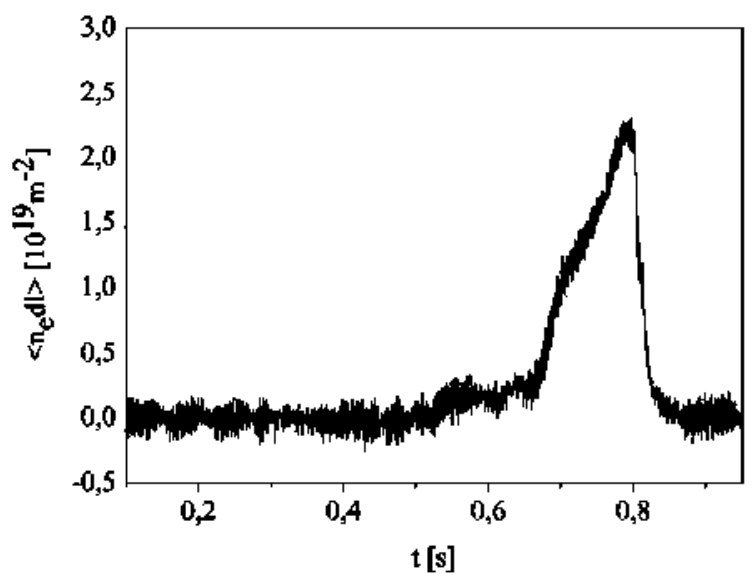

Fig. 4. Elect ronic line average electronic density, zero cross algotithm averaging by 256.

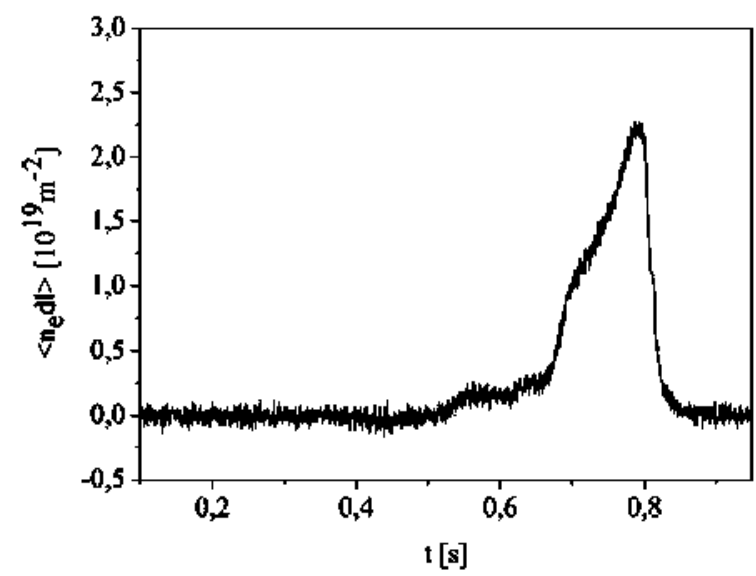

Fig. 5. Electronic line average electronic density, interpolation algorithm averaging by 256 .

processor is going to work. The two approaches have been made, firstly the interpolation has been obviated and the density has been calculated just taking into account the zero crossings and averaging by 256 , with this scheme an rms value of $1 / 125$ of $\mathrm{CO}_{2}$ fringe (1 fringe $\simeq 10^{20} \mathrm{~m}^{-2}$ ) of the density signal is obtained, results can be seen in Fig. 4.

The second approach is to include the interpolation scheme explained in the previous section, in this case and under the same

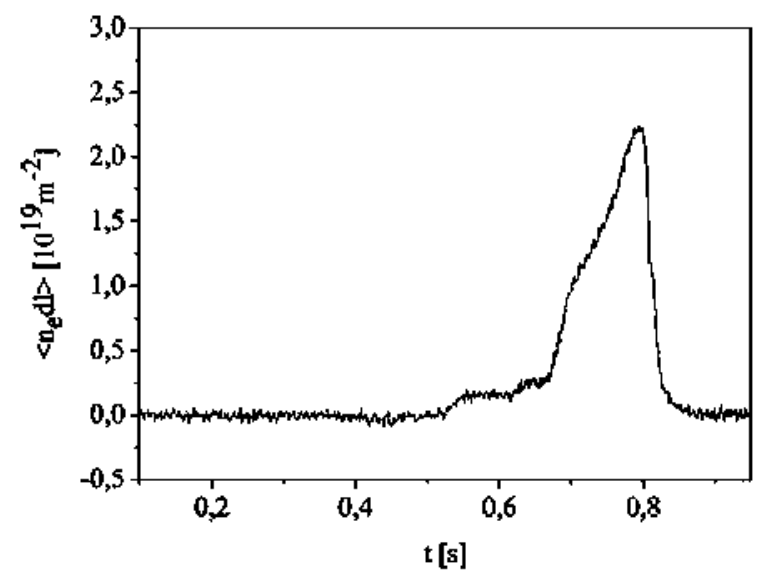

Fig. 6. Electronic line average electronic density, interpolation algorithm ave raging by 1024 . 


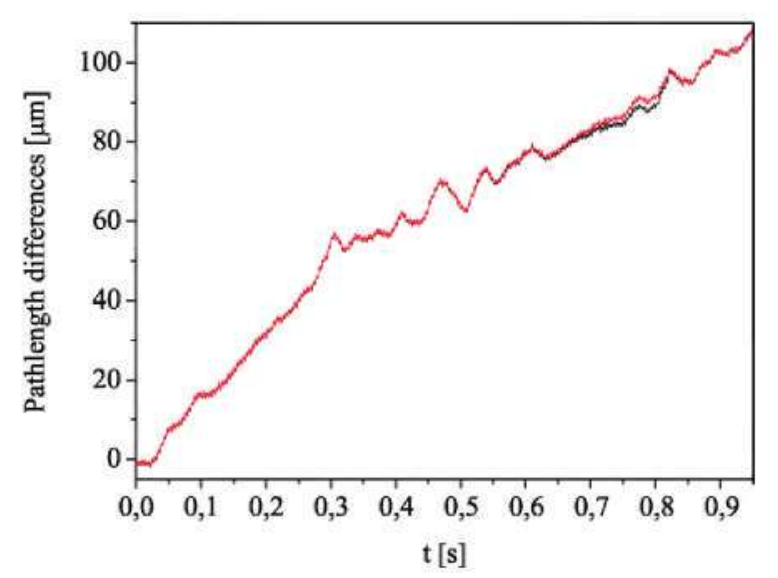

Fig. 7. Path length separation.

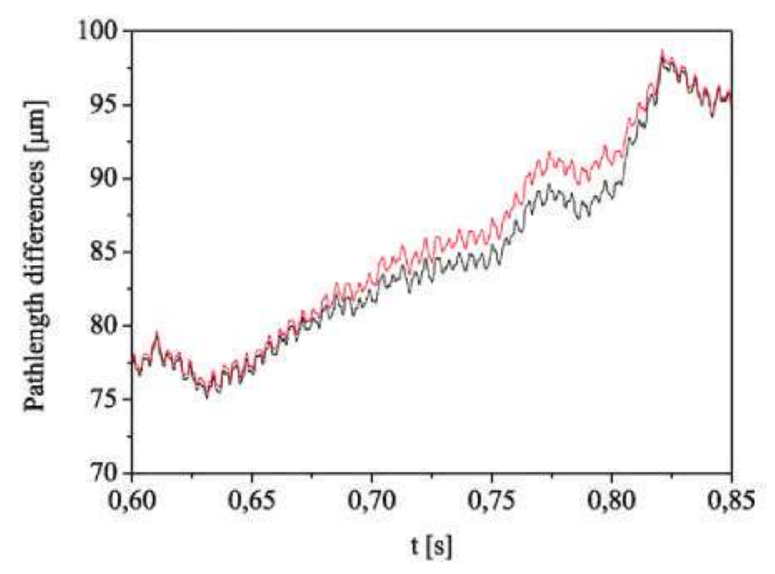

Fig. 8. Path length separation detail.

conditions the quality of the density signal improves significantly, the rms value obtained is $1 / 200$ of $\mathrm{CO}_{2}$ fringe Fig. 5 . In both cases the output velocity is in the range of $200 \mathrm{ksps}$. With few samples of the input signals per period the use of the interpolation algorithm is essential.

Density resolution can be improved reducing data flow, for instance an average of 1024 would give a data flow of $50 \mathrm{ksps}$ and an rms value of $1 / 400$ of $\mathrm{CO}_{2}$ fringe, equivalent to the current postprocessing system but with a sampling rate 10 times higher Fig. 6 . Perhaps improving the quality of the input signals to the system would permit less averaging of the density signals and by hence better resolution [9], interpolation algorithm reduces the product averaging by bandwidth or what is equivalent the product of noise by output time rate.

To achieve a final output resolution in the electron density computation (1) of about $0.5 \times 10^{17} \mathrm{~m}^{-2}$, both phase difference signals $\Delta \varphi_{\mathrm{NdYAC}}$ and $\Delta \varphi_{\mathrm{CO}}$ should be averaged by a factor of 1000 . In Fig. 7 we can see the $\lambda_{\text {NdYAG }} \Delta \varphi_{\text {NdYAG }}$ and $\lambda_{\mathrm{CO}_{2}} \Delta \varphi_{\mathrm{CO}_{2}}$ signals. Both traces are superposed until they began to separate because of plasma density. This separation can be seen in better detail in Fig. 8. The electron density is computed finally subtracting these two traces. The slopes observed in 7 appear mainly because of mechanical movements and vibrations of the machine, this is the reason because a two-color interferometer is needed for these type of measurements.

\section{Conclusions}

A new electronic density measurement system with online processing capabilities has been developed. An output density signal at $200 \mathrm{ksps}$ and an rms value equivalent to $1 / 200$ fringe has been obtained. The actual system is only capable of seeing densities at frequencies one hundred times lower, so it is expected that with this new system high frequency instability effects in plasmas could be seen so a considerable improvement in the characteristics of the current system is expected. Results obtained in the simulations are very good so real results obtained with the synthesized design in the FPGA will be published. One of the main advantages of this new system is that with one unique board all the channels of the future multi-channel interferometer can be acquired at the same time and all the computations are made in parallel. In TJ-II the immediate applications are going to be to control the Thompson Scattering, the gas puffing valves and finally the NBI injection, among others. This system is going to be used also, with several modifications in the W7-X Stellarator IR-interferometer.

\section{References}

[1] C. Alejaldre, et al., Fusion Technol. 17 (1990) 131.

[2] M. Sánchez, T. Estrada, J.Sánchez, P.Acedo, H. Lamela, Rev.Sci. Instrum. 75(2004) 4671.

[3] P. Kornejew, M. Hirsch, T. Bindemann, A. Dinklage, H. Dreier, H. Hartfuß, Rev. Sci. Instrum. 77 (2006), 10F323-1.

[4] M. Sánchez, J. Sánchez, T. Estrada, E. Sánchez, P. Acedo, H. Lamela, Rev. Sci. Instrum. 75 (2004) 10.

[5] M. Sánchez, L. Esteban, P. Kornejew, M. Hirsh. AlP Conference Proceedings 993 , ISBN 978-0-7354-0512-7, 2008.

[6] J.A. López, G. Caffarena, C. Carreras, O. Nieto-Taladriz, IET Circuits, Devices Syst. 2 (2008) 393.

[7] M. Sánchez, J. Sănchez, Rev. Sci. Intrum. 76 (2005) 46104.

[8] K. Bastiaensen, Universiteit Gent, Master Thesis, 2007.

[9] Silicon Laboratories AN118-DS12 Rev 1,2 12/03. 